lustitia Socialis. Revista Arbitrada de Ciencias Jurídicas.

Año V. Vol. V. №3. Edición Especial. 2020-III:

Universidad Regional Autónoma de los Andes

Hecho el depósito de Ley: FA2016000064 ISSN: 2542-3371

FUNDACIÓN KOINONIA (F.K). Santa Ana de Coro, Venezuela

Juan Alberto Rojas-Cárdenas; Daniela Lizeth Aguilar-Barona

http://dx.doi.org/10.35381/racji.v5i3.1099

\title{
La mediación como medio alternativo de solución de conflictos
}

Mediation as an alternative means of conflict resolution

\author{
Juan Alberto Rojas-Cárdenas \\ up.juanrojas@uniandes.edu.ec \\ Universidad Regional Autónoma de los Andes, Puyo \\ Ecuador \\ https://orcid.org/0000-0001-9026-4402 \\ Daniela Lizeth Aguilar-Barona \\ dp.danielalab76@uniandes.edu.ec \\ Universidad Regional Autónoma de los Andes, Puyo \\ Ecuador
}

Recibido: 31 de octubre de 2020

Revisado: 10 de octubre de 2020

Aprobado: 05 de diciembre de 2020

Publicado: 10 de diciembre de 2020 
lustitia Socialis. Revista Arbitrada de Ciencias Jurídicas.

Año V. Vol. V. N³. Edición Especial. 2020-III:

Universidad Regional Autónoma de los Andes

Hecho el depósito de Ley: FA2016000064

ISSN: 2542-3371

FUNDACIÓN KOINONIA (F.K). Santa Ana de Coro, Venezuela

Juan Alberto Rojas-Cárdenas; Daniela Lizeth Aguilar-Barona

\title{
RESUMEN
}

La investigación se trazó como objetivo analizar la mediación como medio alternativo de solución de conflictos en el Ecuador. Se fundamentó metodológicamente desde un tipo descriptiva documental con diseño bibliográfico. Se evidencia que una excesiva burocracia judicial, conduce a casos de corrupción, distorsión de la aplicación de los derechos fundamentales de los grupos sociales vulnerables, requiriéndose una justicia restauradora de los principios éticos de convivencia humana. La cantidad de procesos que ingresan al Centro de Mediación en la provincia de Pastaza es de treinta a cincuenta causas, sin embargo, es una cantidad baja para el numero de causas que se tramitan mediante la justicia tradicional, además las actas de mediación que no se hayan cumplido los acuerdos pactados se demandará su incumplimiento.

Descriptores: Mediación; administración de justicia; procedimiento legal. (Palabras tomadas del Tesauro UNESCO).

\begin{abstract}
The objective of the investigation was to analyze mediation as an alternative means of conflict resolution in Ecuador. It was methodologically based on a descriptive documentary type with bibliographic design. It is evident that excessive judicial bureaucracy leads to cases of corruption, distortion of the application of the fundamental rights of vulnerable social groups, requiring a restorative justice of the ethical principles of human coexistence. The number of processes that enter the Mediation Center in the province of Pastaza is from thirty to fifty cases, however, it is a low number for the number of cases that are processed through traditional justice, in addition to the mediation acts that are not have complied with the agreed agreements, their breach will be demanded.
\end{abstract}

Descriptors: Mediation; administration of justice; legal procedure. (Words taken from the UNESCO Thesaurus). 
lustitia Socialis. Revista Arbitrada de Ciencias Jurídicas.

Año V. Vol. V. N³. Edición Especial. 2020-III:

Universidad Regional Autónoma de los Andes

Hecho el depósito de Ley: FA2016000064

ISSN: 2542-3371

FUNDACIÓN KOINONIA (F.K). Santa Ana de Coro, Venezuela

Juan Alberto Rojas-Cárdenas; Daniela Lizeth Aguilar-Barona

\section{INTRODUCCIÓN}

La mediación se genera como un proceso para articular la solución a un conflicto entre las partes, involucrándose un tercer ente quien hace el rol de mediador para conciliar una solución acordada y concertada, en este sentido, (Rodrígues, 2017), habla de un nuevo paradigma en derecho, donde se transcienda el enfoque punitivo predominante en la sociedad occidental como procedimiento de aplicar justicia, percibiéndose en la mediación, una herramienta para descongestionar el sistema de justicia de procedimientos largos, costosos, para los afectados.

En Chile, la mediación ha sido implementada en los conflictos mercantiles y civiles de carácter patrimonial (Jequier-Lehuedé, 2016), recomendando potenciar su uso, siendo necesario potenciar la buena fe de las partes involucradas, para asegurar un efectivo proceso de mediación, sin embargo, es necesario contar con protocolos jurídicos para regularizar tal acción, por cuanto la buena fe parte de lo subjetivo de las personas.

En Colombia, la mediación ha sido implementada como mecanismo de restauración de la justicia, a través del diálogo con la finalidad de pacificar a la sociedad (Mazo-Álvarez, 2014), proyectándose además la empatía como factor social para comprender la perspectiva de las partes involucradas, en ese sentido, se fomentan valores éticos con la finalidad de propiciar desde el sentido de paz, la construcción de una sociedad encausada por la justicia.

Por otro lado, (Gorjón-Gómez \& Sauceda-Villeda, 2018), presenta la experiencia de la mediación como justicia restaurativa en contextos comunitarios, siendo favorable para la resolución de conflictos, solventándose por el mutuo acuerdo de las partes, evitándose la controversia de asistir a los tribunales en donde se corre el riesgo de prolongar en el tiempo y costo, una decisión por parte del juez en razón de promover la justicia requerida. Sin embargo, (Serrano-Lucero, 2016), advierte que es necesario conocer la esencia del Estado sobre la mediación, es decir, si realmente pretende a través de la legislación verticalidad del poder, o generar mecanismos alternativos para cooperar en la población de menos acceso al logro de una justicia restaurativa sin pasar por la extrema burocracia 
lustitia Socialis. Revista Arbitrada de Ciencias Jurídicas.

Año V. Vol. V. N³. Edición Especial. 2020-III:

Universidad Regional Autónoma de los Andes

Hecho el depósito de Ley: FA2016000064 ISSN: 2542-3371

FUNDACIÓN KOINONIA (F.K). Santa Ana de Coro, Venezuela

Juan Alberto Rojas-Cárdenas; Daniela Lizeth Aguilar-Barona

del sistema judicial tradicional. En este sentido, (Vázquez-Martínez \& Bazán-Mayagoitia, 2019), consideran que se contribuye a la reintegración social, el derecho positivista prosigue en su profundización de violar los derechos humanos, por lo cual, se requiere educar a la ciudadanía sobre el valor de contar con la mediación como proceso judicial para la reedificación de los derechos fundamentales.

Por lo planteado, la investigación se trazó como objetivo analizar la mediación como medio alternativo de solución de conflictos en el Ecuador.

\section{MÉTODO}

La investigación se fundamentó metodológicamente desde un tipo descriptiva documental con diseño bibliográfico, lo cual, permitió a través del método analítico sintético, explorar documentos, leyes, investigaciones, relacionadas al tema, lo cual produjo la descomposición teórica para ser analizada mediante el análisis de contenido jurídico, para construir un postulado teórico emergente desde las partes analizadas.

\section{RESULTADOS}

Tabla 1.

Análisis documental sobre mediación.

\begin{tabular}{|l|l|l|}
\hline \multicolumn{1}{|c|}{ Autor } & \multicolumn{1}{|c|}{ Cita } & \multicolumn{1}{c|}{ Interpretación } \\
\hline Constitución & Artículo 190. el \\
de la República & Se reconoce el arbitraje, la mediación y otros & La mediación en el \\
del Ecuador tiene rango \\
$(2008)$ & procedimientos alternativos para la solución & constitucional, lo \\
& de conflictos. Estos procedimientos se & cual deriva en que se \\
& aplicarán con sujeción a la ley, en materias en & generen leyes en pro \\
& las que por su naturaleza se pueda transigir. & de fomentar esta \\
& En la contratación pública procederá el el & opción como \\
& arbitraje en derecho, previo pronunciamiento & herramienta para la \\
\hline
\end{tabular}


lustitia Socialis. Revista Arbitrada de Ciencias Jurídicas.

Año V. Vol. V. N³. Edición Especial. 2020-III:

Universidad Regional Autónoma de los Andes

Hecho el depósito de Ley: FA2016000064 ISSN: 2542-3371

FUNDACIÓN KOINONIA (F.K). Santa Ana de Coro, Venezuela

Juan Alberto Rojas-Cárdenas; Daniela Lizeth Aguilar-Barona

\begin{tabular}{|c|c|c|}
\hline & $\begin{array}{l}\text { favorable de la Procuraduría General del } \\
\text { Estado, conforme a las condiciones } \\
\text { establecidas en la ley. } \\
\text { "Art } 326 \text {.- El derecho al trabajo se sustenta en } \\
\text { los siguientes principios: } \\
\text { 10. Se adoptará el diálogo social para la } \\
\text { solución de conflictos de trabajo y formulación } \\
\text { de acuerdos. }\end{array}$ & $\begin{array}{lr}\text { solución } & \text { de } \\
\text { conflictos, siendo } \\
\text { empleada en el } \\
\text { ámbito laboral y } \\
\text { penal. }\end{array}$ \\
\hline $\begin{array}{l}\text { Código del } \\
\text { trabajo }\end{array}$ & $\begin{array}{l}\text { Art. 555.- De sus funciones. - Corresponde a } \\
\text { la Dirección y Subdirecciones de Mediación } \\
\text { Laboral. } \\
\text { Art. } 470 \text {.- Mediación obligatoria.- Si no hubiere } \\
\text { contestación o si ésta no fuere enteramente } \\
\text { favorable a las peticiones de los trabajadores, } \\
\text { el inspector del trabajo remitirá todo lo actuado } \\
\text { a la Dirección o Subdirección de Mediación } \\
\text { Laboral respectiva, para que a través de sus } \\
\text { funcionarios convoque a las partes cuantas } \\
\text { veces considere necesarias, con veinte y } \\
\text { cuatro horas de anticipación por lo menos, a } \\
\text { fin de que procuren superar las diferencias } \\
\text { existentes, dentro del término de quince días } \\
\text { contados desde la fecha de inicio de su } \\
\text { intervención. Este término podrá ampliarse a } \\
\text { petición conjunta de las partes. }\end{array}$ & $\begin{array}{l}\text { Se promueve la } \\
\text { mediación como } \\
\text { acción esencial para } \\
\text { lograr acuerdos } \\
\text { entre el patrón y } \\
\text { trabajadores, así se } \\
\text { prevé la negociación } \\
\text { para evitar conflictos } \\
\text { referidos a la } \\
\text { seguridad social del } \\
\text { trabajador. } \\
\text { Se cuenta con el } \\
\text { mecanismo de las } \\
\text { direcciones de } \\
\text { mediación para que } \\
\text { las personas e } \\
\text { instituciones acudan } \\
\text { a solicitar el arbitraje } \\
\text { ante un proceso de } \\
\text { negociación. }\end{array}$ \\
\hline
\end{tabular}


lustitia Socialis. Revista Arbitrada de Ciencias Jurídicas.

Año V. Vol. V. N³. Edición Especial. 2020-III:

Universidad Regional Autónoma de los Andes

Hecho el depósito de Ley: FA2016000064 ISSN: 2542-3371

FUNDACIÓN KOINONIA (F.K). Santa Ana de Coro, Venezuela

Juan Alberto Rojas-Cárdenas; Daniela Lizeth Aguilar-Barona

\begin{tabular}{|c|c|c|}
\hline $\begin{array}{l}\text { Código } \\
\text { Orgánico } \\
\text { Integral Penal }\end{array}$ & $\begin{array}{l}\text { Artículo } 662 \\
\text { El método alternativo de solución de conflictos } \\
\text { se regirá por los principios generales } \\
\text { determinados en este Código y en particular } \\
\text { por las siguientes reglas: } \\
\text { Consentimiento libre y voluntario de la víctima, } \\
\text { del procesado. Tanto la víctima como el } \\
\text { procesado podrán retirar este consentimiento } \\
\text { en cualquier momento de la actuación. } \\
\text { Los acuerdos que se alcancen deberán } \\
\text { contener obligaciones razonables y } \\
\text { proporcionadas con el daño ocasionado y la } \\
\text { infracción. } \\
\text { La participación del procesado no se podrá } \\
\text { utilizar como prueba de admisión de } \\
\text { culpabilidad en procedimientos jurídicos } \\
\text { ulteriores. } \\
\text { El incumplimiento de un acuerdo no podrá ser } \\
\text { utilizado como fundamento para una condena } \\
\text { o para la agravación de la pena. } \\
\text { Los facilitadores deberán desempeñar sus } \\
\text { funciones de manera imparcial y velar porque } \\
\text { la víctima y el procesado actúen con mutuo } \\
\text { respeto. } \\
\text { La víctima y el procesado tendrán derecho a } \\
\text { consultar a una o un defensor público o } \\
\text { privado." }\end{array}$ & $\begin{array}{l}\text { En lo penal, se } \\
\text { genera la mediación } \\
\text { con la finalidad de } \\
\text { promover la justicia } \\
\text { restaurativa como } \\
\text { alternativa al } \\
\text { derecho positivista, } \\
\text { el cual, } \\
\text { tradicionalmente se } \\
\text { basa en lo punitivo } \\
\text { para castigar una } \\
\text { acción, la cual en } \\
\text { ocasiones pasa por } \\
\text { un largo proceso } \\
\text { judicial, siendo esto } \\
\text { lo que procura evitar } \\
\text { la mediación entre } \\
\text { las partes. }\end{array}$ \\
\hline
\end{tabular}


lustitia Socialis. Revista Arbitrada de Ciencias Jurídicas.

Año V. Vol. V. N³. Edición Especial. 2020-III:

Universidad Regional Autónoma de los Andes

Hecho el depósito de Ley: FA2016000064 ISSN: 2542-3371

FUNDACIÓN KOINONIA (F.K). Santa Ana de Coro, Venezuela

Juan Alberto Rojas-Cárdenas; Daniela Lizeth Aguilar-Barona

\begin{tabular}{|c|c|c|}
\hline $\begin{array}{l}\text { Código de la } \\
\text { Niñez } \\
\text { Adolescencia }\end{array}$ & $\begin{array}{l}\text { Artículos } 345 \text { - } 346 \\
\text { Referidos a la conciliación }\end{array}$ & $\begin{array}{l}\text { Permite a las familias } \\
\text { acudir a un juez de } \\
\text { mediación con la } \\
\text { finalidad de acordar } \\
\text { lo referido al mejor } \\
\text { bien para los } \\
\text { menores, evitándose } \\
\text { largos conflictos } \\
\text { judiciales. }\end{array}$ \\
\hline
\end{tabular}

\section{Causas resueltas correspondiente al año 2017 - 2018}

Es importante destacar que la mediación como solución de conflictos en el centro de mediación de la provincia de Pastaza, durante el año 2017 resolvió un total de 380 expedientes. En cuanto al año 2018 solucionó un total de 294 expedientes mismos que son entregados al archivo general y 8 expedientes están sin pago de tasa final.

En conclusión, se puede evidenciar que en el año 2018 hay una disminución de 11\% de usuarios, pudiendo así evidenciar que la falta de conocimiento a la colectividad sobre el centro de mediación como una herramienta de solución a los conflictos que se puede producir en diferentes ámbitos.

Se evidencia que no existe un número elevado de causas que ingresan al centro de mediación, sin embargo, se lo considera una liberación a la carga laboral para los administradores de justicia de la Provincia de Pastaza.

\section{DISCUSIÓN}

Los resultados evidencian una transición hacia el abordaje del nuevo paradigma jurídico basado en la conciliación, (Entelman, 2005), considera que a pesar de prevalecer el enfoque sacionador en lo penal, la reparación o mediación puede desarrollarse en paralelo al juicio tradicional o hasta evitarlo, lo cual, implica un descongestionaminto de la burocrática justicia, en complemento, (Leal, 2006), comenta que una excesiva burocracia judicial, conduce a casos de corrupción, distorsión de la aplicación de los 
lustitia Socialis. Revista Arbitrada de Ciencias Jurídicas.

Año V. Vol. V. N³. Edición Especial. 2020-III:

Universidad Regional Autónoma de los Andes

Hecho el depósito de Ley: FA2016000064

ISSN: 2542-3371

FUNDACIÓN KOINONIA (F.K). Santa Ana de Coro, Venezuela

Juan Alberto Rojas-Cárdenas; Daniela Lizeth Aguilar-Barona

derechos fundamentales de los grupos sociales vulnerables, requiriéndose una justicia restauradora de los principios éticos de convivencia humana.

Aunado a no contradecir el principio de celeridad (Jarama-Castillo, et al., 2019), el cual involucra una justicia efectiva tanto en aplicación como en proceso, siendo que la mediación se acerca como mecanismo de propiciar una celeridad para la justa restauración de la sociedad, lo cual concuerda con las investigaciones de (JequierLehuedé, 2016), (Mazo-Álvarez, 2014), (Gorjón-Gómez \& Sauceda-Villeda, 2018) y (Vázquez-Martínez \& Bazán-Mayagoitia, 2019), quienes han estudiado la aplicación de la mediación en diversos contextos sociales, confirmándose su aporte a una asertiva justicia social.

\section{CONCLUSIONES}

La mediación es un procedimiento voluntario eficaz, con el cual se puede resolver una gran cantidad de conflictos, ya que, a diferencia de la forma de justicia tradicional, este método alternativo agilita el proceso de solución de un conflicto, además que, es asistido por un mediador imparcial, por lo que no existe ningún tipo de favoritismo pues las partes son las que se ponen sus condiciones.

La cantidad de procesos que ingresan al Centro de Mediación en la provincia de Pastaza es de treinta a cincuenta causas, sin embargo, es una cantidad baja para el numero de causas que se tramitan mediante la justicia tradicional, además las actas de mediación que no se hayan cumplido los acuerdos pactados se demandará su incumplimiento.

\section{FINANCIAMIENTO}

No monetario.

\section{AGRADECIMIENTO}

A la Universidad Regional Autónoma de Los Andes; por motivar el desarrollo de la Investigación. 
lustitia Socialis. Revista Arbitrada de Ciencias Jurídicas.

Año V. Vol. V. N³. Edición Especial. 2020-III:

Universidad Regional Autónoma de los Andes

Hecho el depósito de Ley: FA2016000064 ISSN: 2542-3371

FUNDACIÓN KOINONIA (F.K). Santa Ana de Coro, Venezuela

Juan Alberto Rojas-Cárdenas; Daniela Lizeth Aguilar-Barona

\section{REFERENCIAS CONSULTADAS}

Código de la Niñez y Adolescencia. Ley No. 2002-100. Recuperado de https://n9.cl/fokqg

Código del trabajo. Registro Oficial Suplemento 167 de 16-dic-2005. Última modificación: 26-sep-2012. Recuperado de https://n9.cl/z3p5n

Código Orgánico Integral Penal. Suplemento -- Registro Oficial № 180 -- Lunes 10 de febrero de 2014. Recuperado de https://n9.cl/g6sc

Constitución de la República del Ecuador (2008). Sección octava Medios alternativos de solución de conflictos. Recuperado de https://n9.cl/rp6p

Entelman, R. (2005). Teoria del conflicto . Barcelona España: Gedisa S.A.

Gorjón-Gómez, G, \& Sauceda-Villeda, B. (2018). Justicia restaurativa, una herramienta de paz en la resolución de conflictos comunitarios. Caso Nuevo León. [Restorative justice, a tool of peace in the resolution of community conflicts. Nuevo León case]. Política criminal, 13(25), 548-571. https://dx.doi.org/10.4067/S0718$\underline{33992018000100548}$

Jarama-Castillo, Z, Vásquez-Chávez, J, \& Durán-Ocampo, A. (2019). El principio de celeridad en el código orgánico general de procesos, consecuencias en la audiencia. [The principle of celerity in the general organic code of processes, consequences in the audience]. Revista Universidad y Sociedad, 11(1), 314-323.

Jequier-Lehuedé, E. (2016). La mediación como alternativa de solución de los conflictos empresariales en Chile: Razones y mecanismos para su regulación. [The mediation like an alternative for the solution of enterprise conflicts in Chile. Reasons and mechanisms for his regulation]. Revista de derecho (Valdivia), 29(1), 91-118. https://dx.doi.org/10.4067/S0718-09502016000100005

Leal, A. (2006). Burocracia, justicia y pluralismo jurídico. Una exploración de los espacios del poder en Oaxaca. [Bureaucracy, justice and legal pluralism: an exploration concerning spaces of power in Oaxaca]. Alteridades, 16(31), 39-48.

Mazo-Álvarez, H. M. (2014). La mediación como herramienta de la justicia restaurativa. [Mediation as a tool for restorative justice]. Opinión Jurídica, 12(23). 
lustitia Socialis. Revista Arbitrada de Ciencias Jurídicas.

Año V. Vol. V. N³. Edición Especial. 2020-III:

Universidad Regional Autónoma de los Andes

Hecho el depósito de Ley: FA2016000064 ISSN: 2542-3371

FUNDACIÓN KOINONIA (F.K). Santa Ana de Coro, Venezuela

Juan Alberto Rojas-Cárdenas; Daniela Lizeth Aguilar-Barona

Rodrígues, C. (2017). La Mediación. ¿Una respuesta al nuevo paradigma del Derecho? [Mediation. An answer to the new paradigm of Law?]. Revista de Derecho (Universidad Católica Dámaso A. Larrañaga, Facultad de Derecho), (15), 243256. https://dx.doi.org/10.22235/rd.v1115.1382

Serrano-Lucero, C. (2016). Justicia restaurativa: la desatinada prohibición de la mediación penal en los asuntos de violencia de género. [Restorative Justice: The Foolish Ban on Criminal Mediation in Gender Violence Matters]. CAPJuridica, 1(1), 147-178.

Vázquez-Martínez, A, \& Bazán-Mayagoitia, N. (2019). Justicia restaurativa y reintegración social: retos procedimentales y estructurales. [Restorative Justice and Social Reintegration: Procedural and Structural Challenges]. URVIO Revista Latinoamericana de Estudios de Seguridad, (24), 98113. https://doi.org/10.17141/urvio.24.2019.3789 